\section{CAMRELIZUMAB AND APATINIB COMBINED WITH CHEMOTHERAPY (MFOLFOX6) AS NEOADJUVANT THERAPY FOR LOCALLY ADVANCED RIGHT-SIDED COLON CANCER (AMBITION)}

Zhou Tong*, Sen Lu, Xiaomeng Dai, Xiaobin Cheng, Xuanwen Bao, Xudong Zhu, Xiaofei Cheng, Qihan Fu, Danyang Wang, Hangyu Zhang, Qinsong Sheng, Lulu Liu, Guoliang Zhang, Yi Zheng, Fanlong Liu, Peng Zhao, Wenbin Chen, Weijia Fang, Xiangmin Xu. The First Affiliated Hospital, Zhejiang University School of Medicine, Hangzhou, China

Background Colorectal cancer is a heterogeneous disease with complicated genetic alterations. Right colon and left colon have different features while right colon cancer displays an even worse prognosis. The randomized phase III FOxTROT trial demonstrated better downstaging effect with neoadjuvant plus adjuvant chemotherapy compared with adjuvant chemotherapy alone $(\mathrm{P}=0.04) .{ }^{1}$ Moreover, 2-year relapse rate was improved with neoadjuvant therapy, though the difference was not statistically significant. The NICHE study of neoadjuvant immunotherapy (maximum 6 weeks) showed that the pathological response was observed in 20/20 mismatch repair-deficient (dMMR) resectable colon cancers, with 19 major pathological responses and 12 pathological complete responses (pCRs). ${ }^{2}$ Recently, KEYNOTE-177 study showed improved progression-free survival with PD-1 inhibitor over chemotherapy (16.5 months vs. 8.2 months) in untreated microsatellite instability-high (MSI-H)/dMMR colon cancer patients, including $68 \%$ of right colon cancers. ${ }^{3}$ In addition, camrelizumab (PD-1 inhibitor) plus apatinib (vascular endothelial growth factor receptor-2 tyrosine kinase inhibitor) demonstrated favorable antitumor effects and a manageable safety profile in advanced hepatocellular carcinoma and gastric cancer. ${ }^{4} 5$ This phase II trial aims to explore whether the combination of camrelizumab, apatinib and chemotherapy (mFOLFOX6) could significantly improve the pathological regression rate in locally advanced right colon cancer so as to bring considerable survival benefit for patients.

Methods Eligible patients are aged 18-75 years, with locally advanced (T4 or T3 with extramural depth $\geq 5 \mathrm{~mm}$, N0-2, M0, AJCC 8th) adenocarcinoma of right colon (including ileocecal area, ascending colon, and transverse colon to splenic flexion), and without prior systemic chemotherapy or immunotherapy. All patients will receive 5 cycles of camrelizumab (200 mg once every 2 weeks) plus mFOLFOX6 and 2 months of apatinib (250 mg orally once a day), followed by surgery and 7 cycles of adjuvant camrelizumab plus mFOLFOX6. The primary endpoint is the proportion of patients with tumor regression grade (TRG) 2-4 according to the Dworak criteria (TRG2: dominantly fibrotic changes with few tumor cells or groups; TRG3: very few tumor cells in fibrotic tissue; TRG4: no tumor cells). Secondary endpoints include downstaging rate, $\mathrm{pCR}$ rate, $\mathrm{R} 0$ resection rate, 2 -year disease-free survival rate, 2-year event-free survival, overall survival, quality of life, and safety.

Results To date, three of planned 64 patients have been enrolled. Two patients have completed surgery. According to Dworak criteria, TRG ranked 4 (pathologic complete response) for the first patient and 3 (very few tumor cells in fibrotic tissue) for the second patient. No severe adverse events have been observed for all patients.

Trial Registration This trial has been registered at ClinicalTrials.gov (NCT04625803).

\section{REFERENCES}

1.. G. Foxtrot Collaborative. Feasibility of preoperative chemotherapy for locally advanced, operable colon cancer: the pilot phase of a randomised controlled trial. Lancet Oncol 13(11) (2012):1152-60.

2.. Chalabi M, Fanchi LF, Dijkstra KK, Van den Berg JG, Aalbers AG, Sikorska K, Lopez-Yurda M, Grootscholten C, Beets GL, Snaebjornsson P, Maas M, Mertz M, Veninga $V$, Bounova $G$, Broeks $A$, Beets-Tan RG, de Wijkerslooth TR, van Lent AU, Marsman HA, Nuijten E, Kok NF, Kuiper M, Verbeek WH, Kok M, Van Leerdam ME, Schumacher TN, Voest EE, Haanen JB. Neoadjuvant immunotherapy leads to pathological responses in MMR-proficient and MMR-deficient early-stage colon cancers. Nat Med 26(4) (2020):566-576.

3.. André $T$, Shiu KK, Kim TW, Jensen BV, Jensen $L H$, Punt $C$, Smith $D$, Garcia-Carbonero R, Benavides M, Gibbs P, de la Fouchardiere C, Rivera F, Elez E, Bendell J, Le DT, Yoshino T, Van Cutsem E, Yang P, Farooqui MZH, Marinello P, Diaz Jr LA. Pembrolizumab in microsatellite-instability-high advanced colorectal cancer. $N$ Engl J Med 383(23) (2020):2207-2218

4.. Xu J, Shen J, Gu S, Zhang Y, Wu L, Wu J, Shao G, Zhang Y, Xu L, Yin T, Liu J, Ren Z, Xiong J, Mao X, Zhang L, Yang J, Li L, Chen X, Wang Z, Gu K, Chen X, Pan Z, Ma K, Zhou X, Yu Z, Li E, Yin G, Zhang X, Wang S, Wang Q. Camrelizumab in combination with apatinib in patients with advanced hepatocellular carcinoma (RESCUE): a nonrandomized, open-label, phase II trial. Clin Cancer Res 27 (4) (2021):1003-1011.

5.. Xu J, Shen J, Gu S, Zhang Y, Wu L, Wu J, Shao G, Zhang Y, Xu L, Yin T, Liu J, Ren Z, Xiong J, Mao X, Zhang L, Yang J, Li L, Chen X, Wang Z, Gu K, Chen X, Pan Z, Ma K, Zhou X, Yu Z, Li E, Yin G, Zhang X, Wang S, Wang Q, Xu J, Zhang $Y$, Jia R, Yue C, Chang L, Liu R, Zhang G, Zhao C, Zhang Y, Chen C, Wang Y, Yi X, Hu Z, Zou J, Wang Q. Camrelizumab in combination with apatinib in patients with advanced hepatocellular carcinoma (RESCUE): a nonrandomized, open-label, phase II trial anti-PD-1 antibody SHR-1210 combined with apatinib for advanced hepatocellular carcinoma, gastric, or esophagogastric junction cancer: an open-label, dose escalation and expansion study. Clin Cancer Res 27(4) (2021):1003-1011.

Ethics Approval Study protocol was approved by the Clinical Research Ethics Committee of the First Affiliated Hospital, College of Medicine, Zhejiang University (2020-119)

Consent Written informed consent was obtained from the patient for publication of this abstract and any accompanying images. A copy of the written consent is available for review by the Editor of this journal.

http://dx.doi.org/10.1136/jitc-2021-SITC2021.412 